\title{
Integrated Network Pharmacology and Experimental Validation Approach to Investigate the Therapeutic Effects of Capsaicin on Lipopolysaccharide-Induced Acute Lung Injury
}

\author{
Peihui Liu, ${ }^{1}$ Jindou Hao, ${ }^{1}$ Jie Zhao, ${ }^{2}$ Rong Zou, ${ }^{3}$ Juan Han, ${ }^{1}$ Jia Tian, ${ }^{1}$ Wanqu Liu, ${ }^{1}$ \\ and Hao Wang ${ }^{4}$ \\ ${ }^{1}$ Department of Pediatrics, Affiliated Shenzhen Maternity \& Child Healthcare Hospital, Southern Medical University, Shenzhen, \\ Guangdong, China \\ ${ }^{2}$ Department of Neonatology, Affiliated Shenzhen Maternity \& Child Healthcare Hospital, Southern Medical University, Shenzhen, \\ Guangdong, China \\ ${ }^{3}$ Department of Pharmacy, Affiliated Shenzhen Maternity \& Child Healthcare Hospital, Southern Medical University, Shenzhen, \\ Guangdong, China \\ ${ }^{4}$ Affiliated Shenzhen Maternity \& Child Healthcare Hospital, Southern Medical University, Shenzhen, Guangdong, China
}

Correspondence should be addressed to Hao Wang; wangh2110@163.com

Received 3 October 2021; Revised 18 December 2021; Accepted 31 December 2021; Published 29 January 2022

Academic Editor: Joilson O. Martins

Copyright (c) 2022 Peihui Liu et al. This is an open access article distributed under the Creative Commons Attribution License, which permits unrestricted use, distribution, and reproduction in any medium, provided the original work is properly cited.

\begin{abstract}
An integrated method combining network pharmacology and in vivo experiment was performed to investigate the therapeutic mechanism of capsaicin (Cap) against acute lung injury. The potential key genes and signaling pathways involved in the therapeutic effect of Cap were predicted by the network pharmacology analyses. Additionally, the histological assessment, ELISA, and RT-qPCR were performed to confirm the therapeutic effect and the potential mechanism action involved. Our findings showed that TNF, IL-6, CXCL1, CXCL2, and CXCL10 were part of the top 50 genes. Enrichment analysis revealed that those potential genes were enriched in the TNF signaling pathway and IL-17 signaling pathway. In vivo experiment results showed that Cap alleviated histopathological changes, decreased inflammatory infiltrated cells and inflammatory cytokines, and improved antioxidative enzyme activities in the bronchoalveolar lavage fluid (BALF). Furthermore, Cap treatment effectively downregulated TNF, IL-6, NF- $\kappa$ B, CXCL1, CXCL2, and CXCL10 in lung tissue. Thus, our findings demonstrated that Cap has the therapeutic effect on LPS-induced acute lung injury in neonatal rats via suppression of the TNF signaling pathway and IL-17 signaling pathway.
\end{abstract}

\section{Introduction}

Acute lung injury is the initial stage of the entire pathological progression of acute respiratory distress syndrome. It is also a life-threatening inflammatory syndrome with high mortality and morbidity [1]. Acute respiratory distress syndrome is the more severe manifestation of acute lung injury, which is characterized by lung inflammation, pulmonary edema, diffuse alveolar damage, and increased neutrophil infiltration $[2,3]$. Newborns, particularly preterm infants, are susceptible to acute lung injury, which is one of the most common causes of neonatal death $[4,5]$. Besides, it has been reported that uncontrolled pulmonary inflammation is involved in the pathological process of acute lung injury in infants [6]. To date, current treatment strategies have not been effective in decreasing the mortality and incidence of acute lung injury [7]. Therefore, it is extremely urgent to develop an effective drug for the treatment of acute lung injury.

Lipopolysaccharide (LPS) is a pathogenic endotoxin of Gram-negative bacteria that promotes the generation of inflammatory cytokines and reactive oxygen species [8]. Previous reports have revealed that administration of bacterial LPS to neonatal rats could cause inflammatory injury of 
the neonatal lung $[9,10]$. Therefore, LPS has been widely used as an inducer of acute lung injury in the establishment of lung injury models $[11,12]$. Several signaling pathways are involved in the pathogenesis of LPS-induced acute lung injury, which is closely related to the regulation of oxidative stress and inflammatory response [8]. Accumulating reports revealed that LPS could trigger pulmonary inflammation via activation of nuclear factor-kappa B (NF- $\kappa \mathrm{B})$, which leads to excessive proinflammatory cytokines such as IL- 6 and TNF- $\alpha$ $[13,14]$. Subsequently, these inflammatory cytokines could further cause endothelial and epithelial cell injury in the lung tissue. Thus, inhibiting pulmonary proinflammatory cytokine levels might be an effective therapeutic strategy for the prevention and treatment of neonatal acute lung injury.

Capsaicin (Cap) is an active ingredient of chili pepper, which has been widely investigated for its various pharmacological activities, including analgesic, anti-inflammatory, antioxidant, circadian modulatory, antilithogenic, cardioprotective, and anticarcinogenic effects $[15,16]$. The previous reports indicated that Cap exerted the chemopreventive effects against lung carcinogenesis via decreasing the expression of inflammatory cytokines [17]. Cap pretreatment could decrease pulmonary arterial hypertension via inhibiting lung inflammation [18]. However, the therapeutic effect of Cap against LPS-induced acute lung injury has not yet been reported.

In the present study, the network pharmacology method was used to predict the potential key genes and signaling pathways of Cap in the treatment of acute lung injury. Besides, we established a newborn rat model of acute lung injury to further verify the therapeutic mechanism of Cap on acute lung injury.

\section{Materials and Methods}

2.1. Prediction of Target Genes of Cap. The keyword "capsaicin" was searched in the PubChem database (https://pubchem .ncbi.nlm.nih.gov/) to download 2D structure. Then, the potential genes of Cap were collected from the SwissTargetPrediction database (http://www.swisstargetprediction.ch/) and CTD database (http://ctdbase.org/).

2.2. Collection of Target Genes of Lung Injury. The keyword "lung injury" was searched in the CTD database (http:// ctdbase.org/) and GeneCards database (http://www.genecards .org) to collect disease-related genes. Then, the duplicate genes between the two databases were integrated, and the collected genes were identified as lung injury-related genes. Finally, the intersection genes of Cap-related genes and lung injuryrelated genes were defined as potential therapeutic genes of Cap against lung injury.

2.3. Construction of the PPI Network. The common genes were imported into the STRING database (https://www .string-db.org/), the confidence score was set up as 0.9 , and the species was set Homo sapiens. Then, the TSV format was downloaded and imported into Cytoscape software (3.8.0). The CytoHubba plugin of Cytoscape was used to screen the key genes of the network.
2.4. Animals. All animal experimental operations were strictly carried out in accordance with the guidelines approved by the Institutional Animal Care and Use Committee of Southern Medical University. Sprague-Dawley neonatal rats (10-16 g body weight and 4-8 days old) were obtained from the Animal Experimental Center of Guangdong Province and housed in a specific pathogen-free environment with standard conditions (55-60\% humidity, $20-22^{\circ} \mathrm{C}$ temperature, and a $12 \mathrm{~h}$ dark/light cycle). All animals were allowed free access to water and food ad libitum.

2.5. Experimental Animal Model and Drug Interventions. All rats were randomly divided into a control group and four experimental groups $(n=8)$ : lipopolysaccharide (LPS) group (intraperitoneal injection of $3 \mathrm{mg} / \mathrm{kg}$ of LPS to induce acute lung injury), LPS $+2 \mathrm{mg} / \mathrm{kg}$ of Cap group, LPS $+4 \mathrm{mg} / \mathrm{kg}$ of Cap group, and LPS $+8 \mathrm{mg} / \mathrm{kg}$ of Cap group. After LPS exposure, the rats of the model group received subcutaneous injections of Cap daily for eight consecutive days. LPS (Escherichia coli 055:B5) was obtained from Sigma-Aldrich, and the dose selection was based on the previous report [10]. The doses of Cap used in the present study were according to the previous report and our preliminary experiment [19]. After the last Cap intervention for $24 \mathrm{~h}$, the neonatal rats were sacrificed via intraperitoneal injection of pentobarbital $(50 \mathrm{mg} / \mathrm{kg})$. Lung tissues were collected and stored at $-80^{\circ} \mathrm{C}$ before analysis.

2.6. Lung Tissue Histological Assessment. Lung tissues were fixed with $4 \%$ paraformaldehyde. The tissue samples were dehydrated using a series of concentrations of ethanol. The dehydrated samples were embedded in paraffin and then sliced into $5 \mu \mathrm{m}$ thick sections. Then, the sections were stained using a hematoxylin-eosin reagent and observed under a microscope.

2.7. Evaluation of Lung Edema. The collected lung tissues were rinsed with ice $0.9 \%$ saline and dried using filter paper. Then, the lung tissues were immediately weighed to obtain the wet weight. The lung tissues were dried by baking at $70^{\circ} \mathrm{C}$ for $48 \mathrm{~h}$ and weighed again to obtain the dry weight. Finally, the value of wet weight/dry weight was calculated to evaluate the lung edema.

2.8. Measurement of Inflammatory Infiltrated Cells and Inflammatory Cytokines in the Bronchoalveolar Lavage Fluid (BALF). After the experiment, the BALF was prepared based on the previous study [20]. BALF was centrifuged at $1,500 \mathrm{~g}$ for $15 \mathrm{~min}$ at $4^{\circ} \mathrm{C}$, and the supernatant was collected to obtain the infiltrated cells. Then, the sediment cells were resuspended with ice $0.9 \%$ saline and stained with WrightGiemsa stain (Jiancheng Bioengineering Institute, China). Cells were counted using a hemocytometer under a microscope. The inflammatory cytokine (TNF- $\alpha$, IL-6, and IL- $1 \beta$ ) levels in BALF were measured using a commercial kit per the manufacturer's protocols (Jiancheng Bioengineering Institute, China).

2.9. Measurement of Protein Concentration, MPO Activity, and Antioxidative Enzyme Activities. The total protein level 


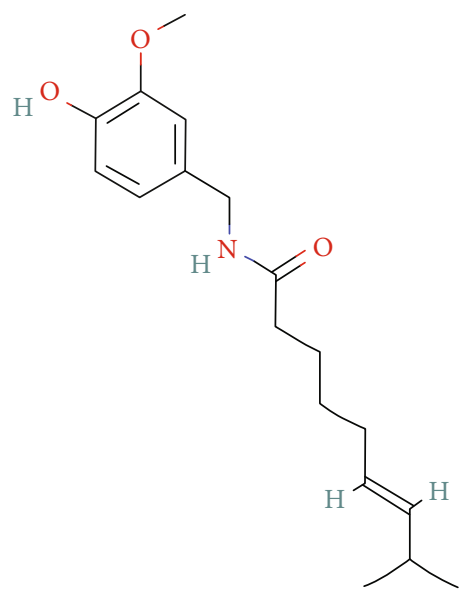

(a)

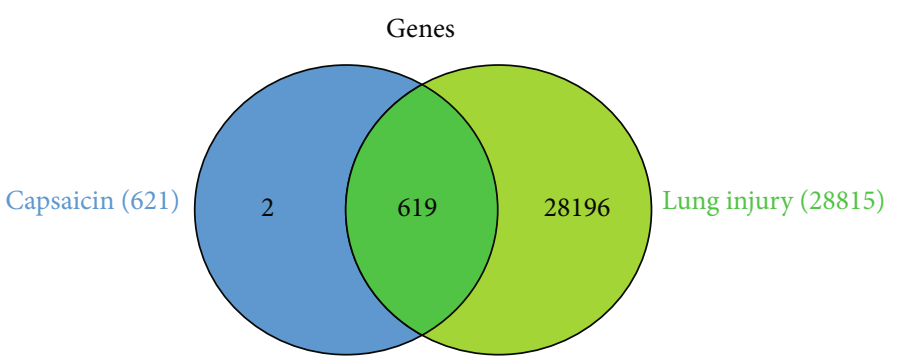

(b)

Figure 1: Potential genes of Cap against acute lung injury. Chemical structure of Cap (a). Venn map between target genes from Cap and lung injury (b).

in the BALF was determined using a bicinchoninic acid protein kit based on the manufacturer's protocols (Jiancheng Bioengineering Institute, China). The lung tissue was homogenized in ice $0.9 \%$ saline and then centrifuged at $10000 \mathrm{~g}$ for $10 \mathrm{~min}$ at $4^{\circ} \mathrm{C}$. The supernatant was collected, and the MPO activity and antioxidative enzyme activities (SOD and GSH) were measured using a commercial kit per the manufacturer's protocols (Jiancheng Bioengineering Institute, China).

2.10. Quantitative RT-PCR. Total RNA of lung tissue was extracted using TRIzol based on the manufacturer's instructions (TIANGEN, USA). $2 \mu \mathrm{g}$ of isolated RNA was reversetranscribed into cDNA using a cDNA synthesis kit (Thermo Fisher, USA) based on the manufacturer's protocols. Then, the qPCR was carried out by using the SYBR GREEN Master Mix (Thermo Fisher, USA) in a LightCycler RT-PCR System (Roche Diagnostics, USA). The $2^{-\Delta \Delta \mathrm{Ct}}$ method was used to quantify the mRNA expression levels. The primers are shown in Supplementary File Table S1.

2.11. Statistical Analysis. Values were expressed as mean \pm standard deviation (SD). The GraphPad 5 software was used to perform the statistical analyses. Statistical significance of the difference between groups was measured by one-way ANOVA followed by Dunnett's multiple comparison test. A $P$ value below 0.05 represents a significant difference.

\section{Results}

3.1. Identification of Potential Targets of Cap. The 2D chemical structure of Cap is presented in Figure 1(a). Based on SwissTargetPrediction and CTD databases, 621 potential genes of Cap were collected after the deletion of duplicate genes.

3.2. Identification and Analysis of Known Acute Lung InjuryRelated Genes. After the deletion of duplicate genes, a total of 28,815 genes were collected from CTD and GeneCards databases. Then, 621 potential genes from Cap were combined with 28,815 acute lung injury-related genes by using a Venn tool to identify the potential genes of Cap against acute lung injury. As shown in Figure 1(b), 619 common genes were identified and could represent the potential genes for acute lung injury treatment with Cap. As shown in Figure 2, the PPI network of the potential genes of Cap acting on acute lung injury was constructed by the STRING database.

3.3. Identification of Inflammation-Related Genes. As shown in Figure 3, the top 50 genes were screened as key targets of Cap against acute lung injury. Most of them were inflammation-related genes, such as CXCL8, CXCR4, CXCL1, CXCL2, IL-6, CXCL10, CCL20, CCL19, IL-2, and TNF. The results showed that inflammatory response might be one of the mechanisms for Cap in the treatment of acute lung injury.

\subsection{Functional Enrichment Analysis of Key Targets. KEGG} and GO enrichment analyses were performed to explore the corresponding pathologic processes of Cap key genes in the treatment of acute lung injury. As shown in Figure 4(a), the top 10 signaling pathways were screened and a bubble map was constructed. In particular, the TNF, IL-6, CXCL1, CXCL2, and CXCL10 were mainly enriched in the inflammation pathways, such as the IL-17 and TNF signaling pathway (Supplementary File Table S2). Therefore, these inflammationrelated genes were selected and verified in the next animal experiment.

As shown in Figure 4(b), the top 10 GO biological processes were screened and a bubble map was constructed. The results showed that multiple biological processes were involved in acute lung injury treatment, such as response to lipopolysaccharide, response to molecule of bacterial origin, response to mechanical stimulus, positive regulation of response to external stimulus, and response to bacterium, which were closely related to these inflammation-related genes.

Based on the findings of the network pharmacology, we further carried out in vivo experiment to validate the therapeutic effect of Cap acting on acute lung injury. 


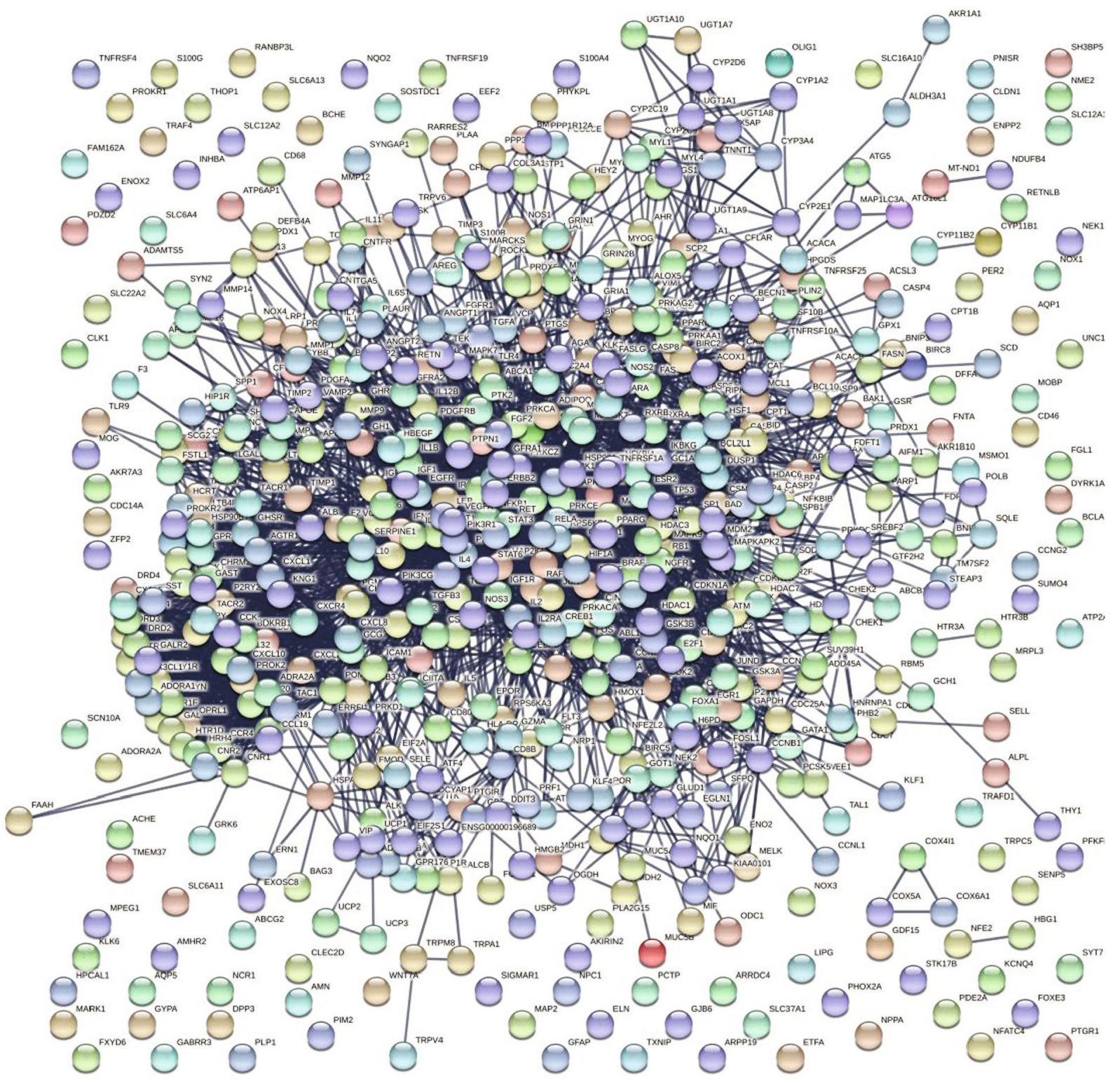

FIgURE 2: Construction of the PPI network for Cap against acute lung injury.

3.5. Cap Alleviated LPS-Induced Acute Lung Injury in Neonatal Rats. H\&E staining was performed to evaluate the protective effect of Cap. As shown in Figure 5(a), the lung tissue in the control group showed normal pulmonary structures. Conversely, neonatal rats from the model group challenged by LPS exhibited clear and significant histopathology alterations, such as alveolar hemorrhage, inflammatory cell infiltration, and alveolar wall edema. However, these pathological features were alleviated to varying degrees by the treatment with Cap ( 4 and $8 \mathrm{mg} / \mathrm{kg}$ ). In particular in the LPS+Cap $(8 \mathrm{mg} / \mathrm{kg})$ group, the alveoli were significantly restored in morphology with mild pathological changes. As shown in Figures 5(b)-5(d), the wet/dry weight ratio, total protein of BALF, and MPO activity were significantly increased after LPS administration compared to those in the control group. All those anomalies were reversed to varying degrees by the treatment with Cap ( 4 and $8 \mathrm{mg} / \mathrm{kg}$ ), especially in the LPS+Cap group. However, a low dose of Cap $(2 \mathrm{mg} / \mathrm{kg})$ does not affect the pulmonary injury in LPStreated neonatal rats.

3.6. Cap Treatment Reduced Inflammatory Cell Accumulation in Neonatal Rats with Acute Lung Injury. LPS stimulation has been reported to induce a strong inflammatory response. In the present study, we measured the levels of inflammatory cell counts in BALF to investigate the underlying protective effects of Cap on lung damage via inhibition of inflammatory accumulation into the alveoli. As shown in Figure 6, the total cells, neutrophils, and macrophages were measured in the control group. Conversely, LPS-exposed neonatal rats showed marked accumulation of total cells, neutrophils, and macrophages, indicating the presence of inflammatory response in the model group. The numbers of infiltrated inflammatory cells were decreased to varying degrees by the treatment with Cap 


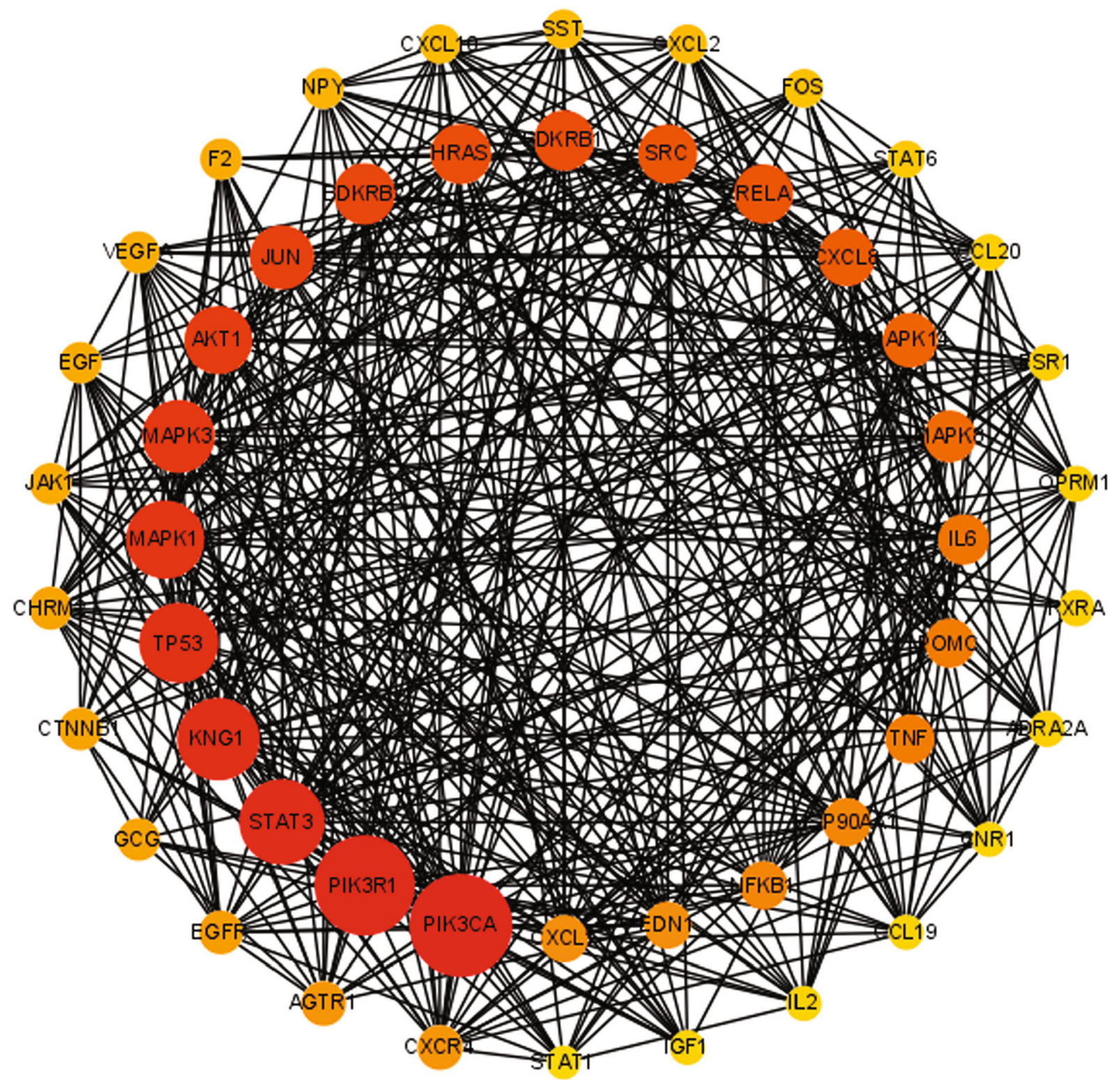

Figure 3: PPI network of top 50 potential genes. As the degree of the target genes increased, the color becomes darker and the circle becomes larger.

(4 and $8 \mathrm{mg} / \mathrm{kg})$, especially in the LPS+Cap $(8 \mathrm{mg} / \mathrm{kg})$ group. However, a low dose of Cap $(2 \mathrm{mg} / \mathrm{kg})$ did not decrease this inflammatory cell accumulation in LPS-treated neonatal rats.

3.7. Cap Treatment Inhibited the Secretion of Inflammatory Cytokines in Neonatal Rats with Acute Lung Injury. In our study, the proinflammatory cytokines in BALF, including TNF- $\alpha$, IL- 6 , and IL- $1 \beta$, were measured by using commercial kits to further demonstrate the anti-inflammatory effects of Cap on neonatal rats with acute lung injury. As shown in Figure 7, in the LPS group, the levels of TNF- $\alpha$, IL-6, and IL- $1 \beta$ were remarkably higher than those in the control group. The levels of inflammatory cytokines were decreased to varying degrees by the treatment with Cap ( 4 and $8 \mathrm{mg} / \mathrm{kg}$ ), especially in the LPS+Cap $(8 \mathrm{mg} / \mathrm{kg})$ group. However, a low dose of
Cap $(2 \mathrm{mg} / \mathrm{kg})$ did not decrease these inflammatory cytokine levels in LPS-treated neonatal rats. These results implied that Cap alleviated LPS-evoked inflammatory response by suppression of inflammatory cell recruitment and decrease in proinflammatory cytokine secretion.

3.8. Cap Treatment Improves Antioxidative Enzyme Activities in Neonatal Rats with Acute Lung Injury. As shown in Figure 8, in the LPS group, the activities of SOD and GSH were remarkably lower than those in the control group. The activities of antioxidative enzymes were improved to varying degrees by the treatment with Cap ( 4 and $8 \mathrm{mg} / \mathrm{kg}$ ), especially in the LPS+Cap $(8 \mathrm{mg} / \mathrm{kg}$ ) group. However, a low dose of Cap $(2 \mathrm{mg} / \mathrm{kg})$ did not improve antioxidative enzyme activities in LPS-treated neonatal rats. Based on these results, the high dose of Cap $(8 \mathrm{mg} / \mathrm{kg})$ was chosen in the next experiment. 


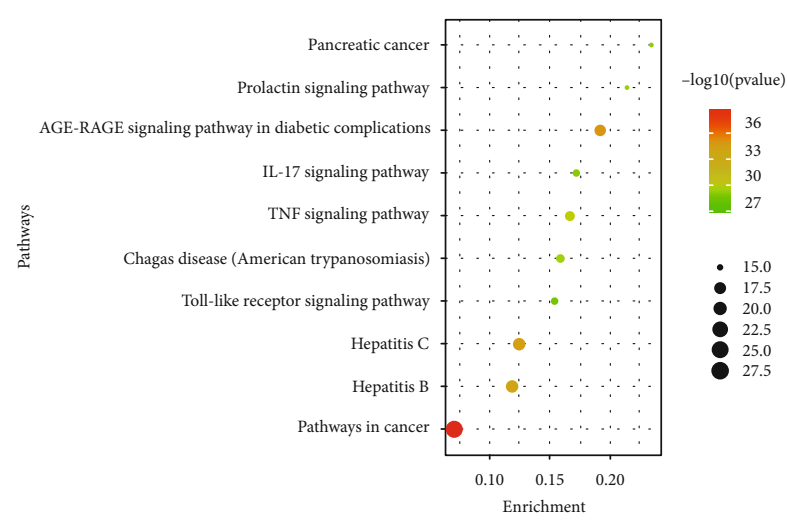

(a)

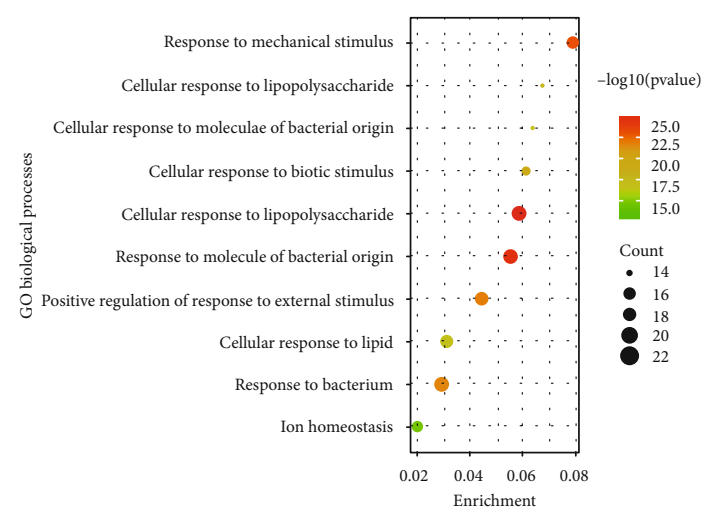

(b)

FIGURE 4: Bubble map of enrichment analysis: (a) KEGG enrichment analysis of Cap against acute lung injury (top 10 were listed); (b) GO enrichment analysis of Cap against acute lung injury (top 10 were listed).
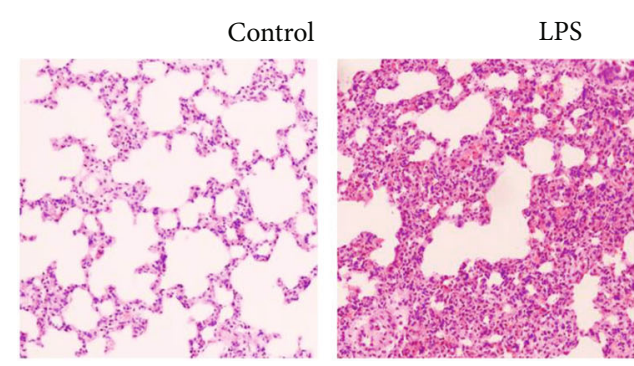

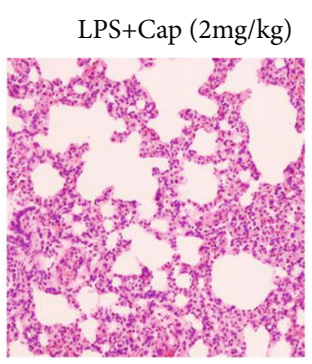

(a)

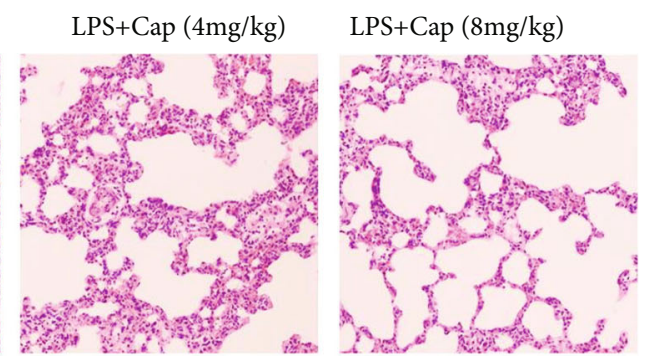

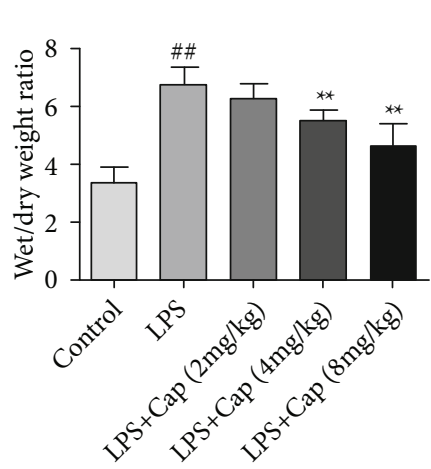

(b)

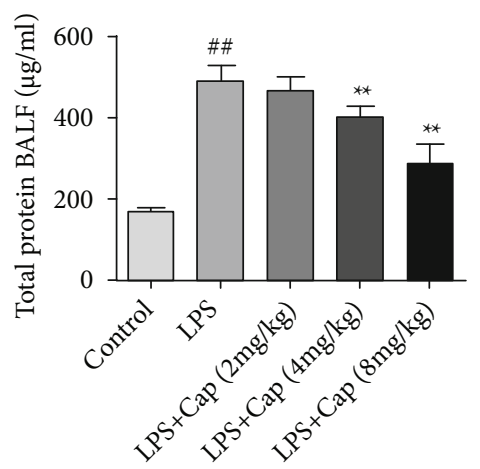

(c)

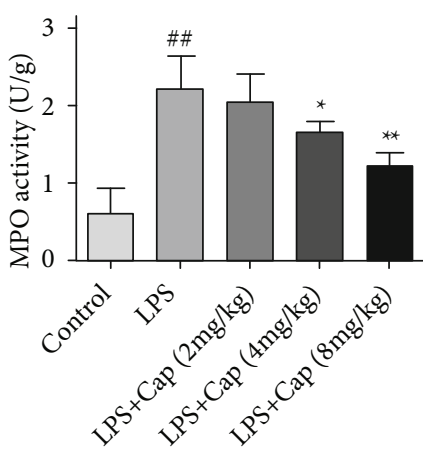

(d)

FIGURE 5: Effects of Cap on experimental pulmonary damage in the acute lung injury animal model. (a) Representative H\&E staining (magnification 200x) of lung tissues in each group. (b) The lung wet/dry weight ratio in each group. (c) Total protein levels in BALF. (d) The activity of MPO in lung tissues. Results are expressed as the mean \pm standard deviation $(n=8)$. ${ }^{\# \#} P<0.01$ compared to the control group; ${ }^{*} P<0.05$ and ${ }^{* *} P<0.01$ compared to the LPS group.

\subsection{Cap Treatment Downregulated Inflammation-Related} Gene Expression in Neonatal Rats with Acute Lung Injury. It has been reported that inflammation was involved in the pathogenesis and progression of acute lung injury [21]. In the present study, the candidate Cap targets (TNF, IL-6, CXCL1, CXCL2, and CXCL10) were identified in the network pharmacology analysis further validated by the qRT-PCR experiment. As shown in Figure 9, in the LPS group, the expression of TNF, IL- $6, \mathrm{NF}-\kappa \mathrm{B}$, CXCL1, CXCL2, and CXCL10 was remarkably higher than that in the control group. Cap treatment downregulated the expression of TNF,
IL-6, NF- $\kappa$ B, CXCL1, CXCL2, and CXCL10 in LPS-treated neonatal rats.

\section{Discussion}

Acute lung injury is an inflammatory disease with a poor prognosis and difficult treatment [22]. The previous report has revealed that acute lung injury pathogenesis is accompanied by functional cell apoptosis, systemic inflammation, and dysfunctions of pulmonary immune homeostasis, which result in endangered life and organ failure [23]. Acute lung 


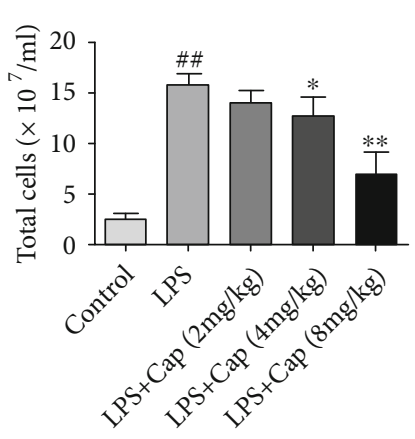

(a)

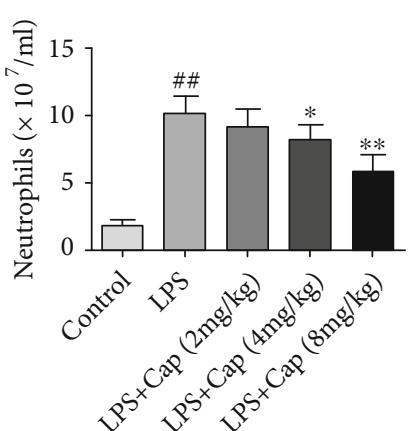

(b)

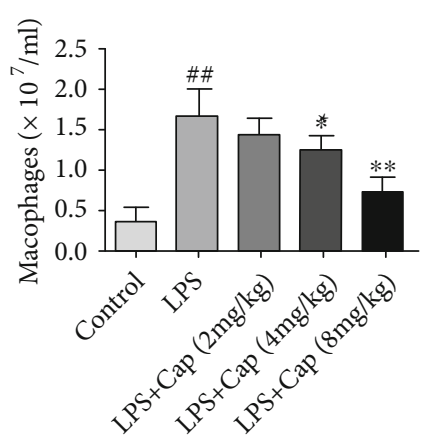

(c)

FIgURE 6: Cap inhibits inflammatory cell accumulation in neonatal rats with acute lung injury. The number of total cells (a), neutrophils (b), and macrophages (c) in BALF. Results are expressed as the mean \pm standard deviation $(n=8) .{ }^{\# \#} P<0.01$ compared to the control group; ${ }^{*} P<0.05$ and ${ }^{* *} P<0.01$ compared to the LPS group.

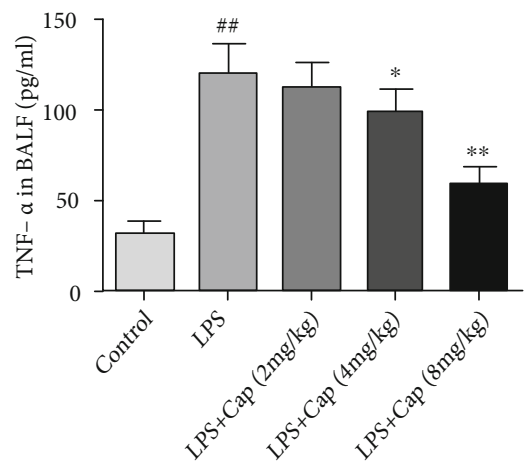

(a)

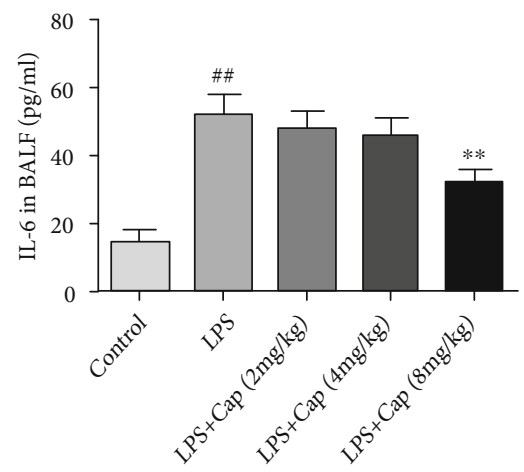

(b)

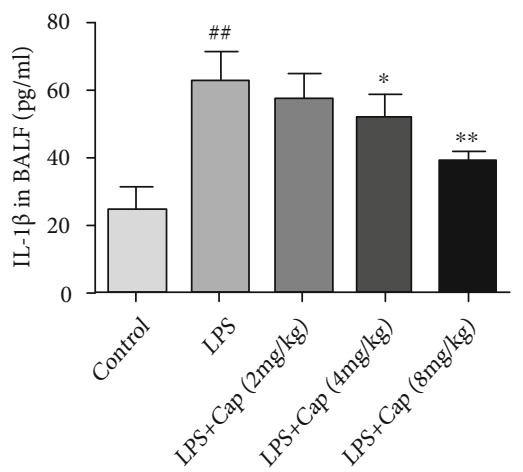

(c)

FIGURE 7: Cap inhibits the release of proinflammatory cytokines in neonatal rats with acute lung injury. The levels of TNF- $\alpha$ (a), IL-6 (b), and IL-1 $\beta$ (c) in BALF. Results are expressed as the mean \pm standard deviation $(n=8) .{ }^{\# \#} P<0.01$ compared to the control group; ${ }^{*} P<0.05$ and ${ }^{* *} P<0.01$ compared to the LPS group.

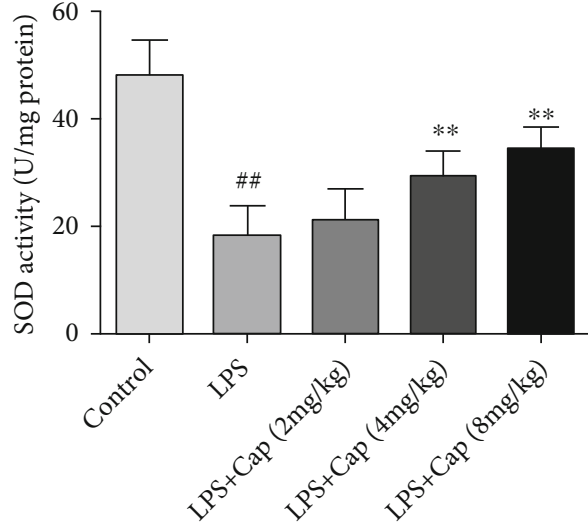

(a)

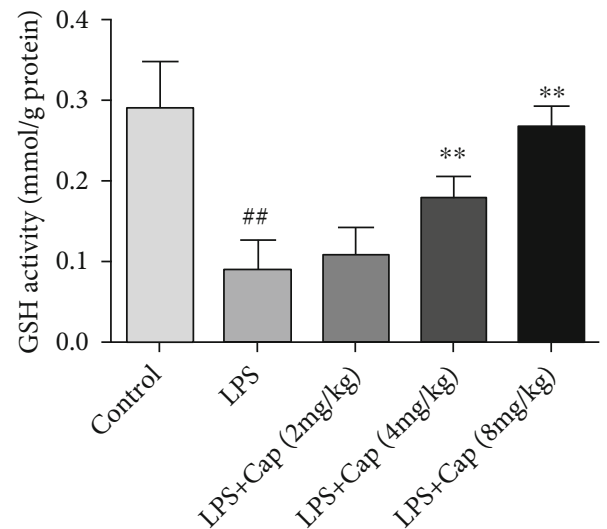

(b)

FIGURE 8: Cap improves the activities of antioxidant enzymes in neonatal rats with acute lung injury. The levels of SOD (a) and GSH (b) in BALF. Results are expressed as the mean \pm standard deviation $(n=8) .{ }^{\# \#} P<0.01$ compared to the control group; ${ }^{* *} P<0.01$ compared to the LPS group.

injury is a refractory respiratory disease with high mortality and high incidence, which brings a burden to society and families. Although some drugs play an anti-inflammatory effect in acute lung injury, these drugs have not been translated into clinical treatment and their use is currently limited to assist in pulmonary disease management $[24,25]$. 


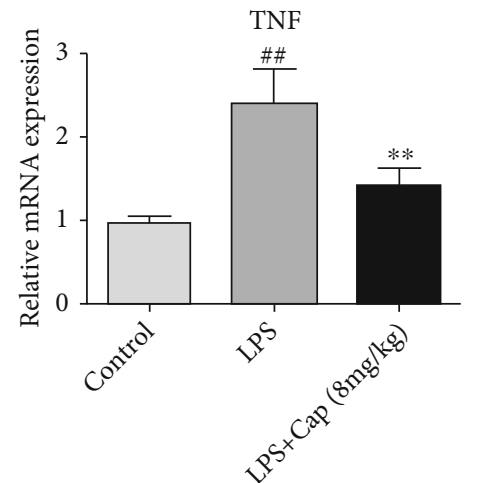

(a)

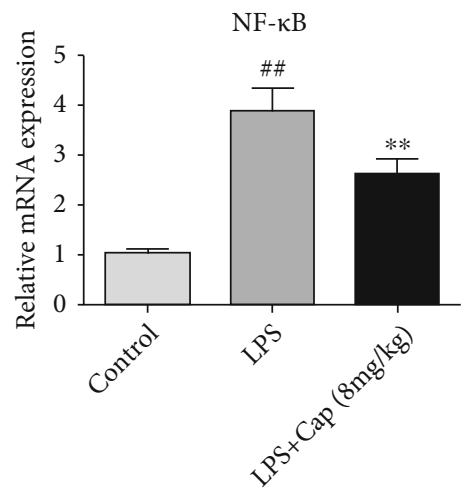

(c)

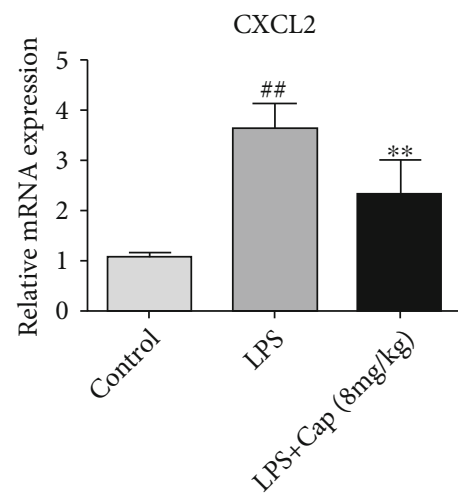

(e)

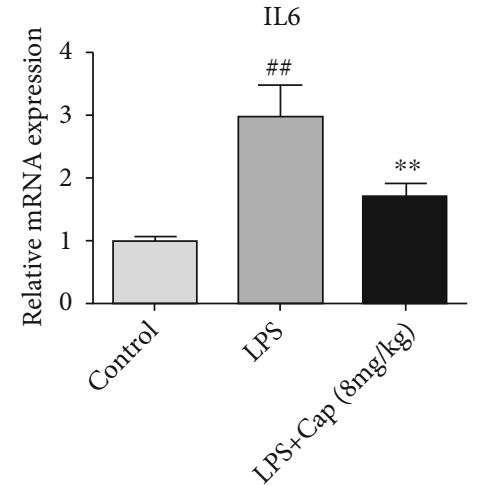

(b)

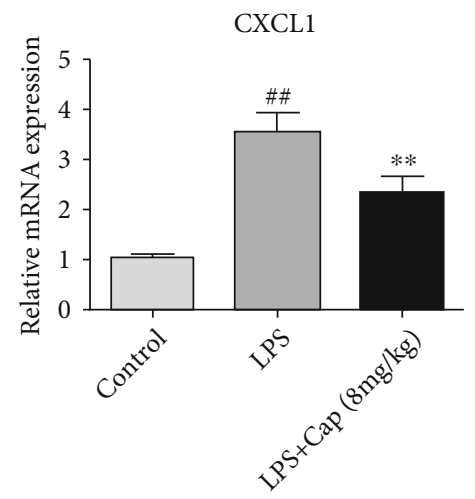

(d)

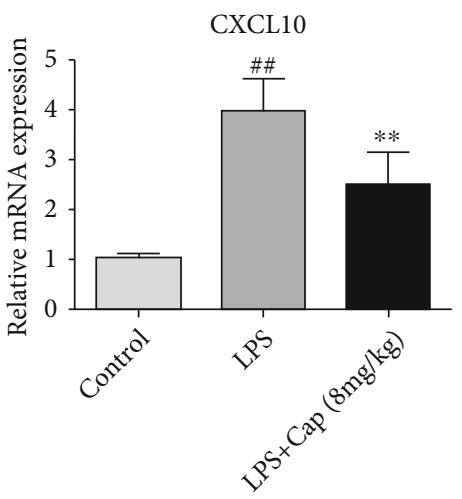

(f)

FIGURE 9: Cap downregulated inflammation-related gene expression in neonatal rats with acute lung injury. The mRNA expression of TNF (a), IL-6 (b), NF- $\kappa$ B (c), CXCL1 (d), CXCL2 (e), and CXCL10 (f) in lung tissue. Results are expressed as the mean \pm standard deviation $(n=6)$. ${ }^{\# \# P} P 0.01$ compared to the control group; ${ }^{*} P<0.05$ and ${ }^{* *} P<0.01$ compared to the LPS group.

Therefore, it is necessary to develop novel and effective therapeutic drugs for acute lung injury treatment.

LPS originates from the cell wall of Gram-negative bacteria and induces tissue damage and edema, excessive production of proinflammatory cytokines, and inflammatory cell infiltration [26]. Therefore, LPS-induced acute lung injury is an ideal and reliable animal model for studying pneumonia-related diseases. Cap is an active ingredient of chili pepper, which has attracted increased attention for its various biological activities $[15,16]$. Cap exerts the chemopreventive effects against lung carcinogenesis via downregulating the expression of inflammatory cytokines [17]. Thus, we speculated that Cap may also have a therapeutic effect on inflammatory pulmonary disease. In the present study, network pharmacology and animal experiment were employed to investigate the therapeutic effect of Cap on acute lung injury.

First, we used the network pharmacology method to screen the potential targets and signaling pathways of Cap in the treatment of acute lung injury. The results of the PPI network revealed that 619 potential targets of Cap have a vital role in acute lung injury treatment, and some inflammatory-related genes, such as CXCL8, CXCR4, CXCL1, CXCL2, IL-6, CXCL10, CCL20, CCL19, IL-2, and 
TNF, were screened out to be closely related to Cap on acute lung injury treatment. Besides, the KEGG enrichment analysis revealed that IL-17 and TNF signaling pathways were the important pathways of Cap against acute lung injury. Next, an animal experiment further confirmed that Cap alleviates lung damage via inhibiting the proinflammatory generation in neonatal rats with acute lung injury.

One of the pathogeneses of acute lung injury is that the imbalance of inflammatory response deteriorates the damage of endothelial or epithelial cells $[27,28]$, which causes an increase in protein levels of the alveoli [29]. Histologically, acute lung injury is characterized by the formation of pulmonary fibrosis, increased alveolar-capillary permeability, a mass of apoptosis in alveolar epithelial cells, and severe acute inflammatory response [30]. In addition, the release of proinflammatory cytokines, excessive neutrophil migration, and destruction of alveolar-capillary membrane integrity are also the major manifestations of acute lung injury cytopathology [31-33]. It has been demonstrated that neutrophils and macrophages are the major sources of multiple inflammatory cytokines in the occurrence and development of lung injury. In response to LPS stimulation, a large number of neutrophils accumulate in the pulmonary capillaries where they are activated, releasing cytotoxic constituents and resulting in damage of pulmonary cells $[34,35]$. A previous report revealed that $\mathrm{T}$ lymphocytes and neutrophils promote the development of pulmonary disease [36]. MPO, a proinflammatory enzyme, is mainly generated by activated neutrophils and it could promote inflammation to worsen and prolong. Previous studies have demonstrated that the MPO activity of the pulmonary tissue is often considered a biomarker of neutrophil infiltration [37]. In the present study, the histopathological changes of pulmonary tissue induced by LPS were well recovered by Cap treatment. The pulmonary edema and MPO activity of neonatal rats with acute lung injury were also alleviated. Besides, the pulmonary inflammation was ameliorated by Cap, as evidenced by decreasing neutrophils and macrophages and inhibiting the secretion of inflammatory cytokines.

A variety of inflammatory mediators are overexpressed in the lung tissue of the acute lung injury model, and TNF and IL-17 signaling pathways were involved in the release of proinflammatory cytokines $[11,38]$. TNF- $\alpha$, a primary proinflammatory mediator in organisms, promotes the activation of macrophages and pulmonary endothelial cells and evokes inflammatory responses [39]. IL-6 is one of the proinflammatory mediators released during acute lung injury and is mainly generated by the innate immune system. Previous reports have revealed that pulmonary-derived IL-6 induces idiopathic pneumonia syndrome through the promotion of Th17 differentiation [40]. In response to LPS stimulation, the generation of chemoattractants CXCL1/ CXCL2 initiates an early phase of neutrophil recruitment [41]. Neutrophils were recruited by CXCL1 into infected tissue to eliminate pathogens in acute lung injury [42]. CXCL2 was mostly overexpressed on resident alveolar macrophages during LPS-induced lung damage [43]. The gene CXCL10 was identified as a potential biomarker for the diagnosis and treatment of acute respiratory distress syndrome [44].
$\mathrm{NF}-\kappa \mathrm{B}$, an upstream transcription factor, evokes lots of genes involved in inflammation, cell adhesion, and immune regulation [45]. The previous report demonstrated that NF$\kappa \mathrm{B}$ activation is implicated in the LPS-induced acute lung injury [46]. Besides, oxidative stress is reconsidered as another inducer that is implicated in the progression of lung injury [47]. It has been reported that LPS treatment evokes the generation of reactive oxygen species and inflammatory cytokines, thus causing acute lung injury in a mouse model [48]. Thus, any ingredient that restrains inflammation and oxidative stress might potentially exert therapeutic effects against lung injury. In this experiment, Cap treatment prevented the secretion of proinflammatory cytokines, improved the activities of antioxidant enzymes, and inhibited the upregulation of TNF, IL-6, NF- $\kappa \mathrm{B}$, CXCL1, CXCL2, and CXCL10 induced by LPS, which demonstrates that the TNF and IL-17 signaling pathways are important for acute lung injury treatment with Cap. Thus, these results demonstrated that Cap effectively blocked the pulmonary inflammation and oxidative stress to control acute lung injury progression and verified the results of network pharmacology.

\section{Conclusions}

In conclusion, the present study preliminarily predicted the potential genes and signaling pathways of Cap alleviating acute lung injury via network pharmacology. Meanwhile, we performed an animal experiment that confirmed the results of network pharmacology. These results provide a scientific basis for the prevention and treatment of acute lung injury.

\section{Data Availability}

The data used to support the study are available from the corresponding author.

\section{Conflicts of Interest}

The authors declared no conflicts of interest.

\section{Supplementary Materials}

Table S1: sequences of primers used in quantitative real-time PCR. Table S2: results of KEGG enrichment analysis. (Supplementary Materials)

\section{References}

[1] L. B. Ware and M. A. Matthay, "The acute respiratory distress syndrome," The New England Journal of Medicine, vol. 342, no. 18, pp. 1334-1349, 2000.

[2] M. A. Matthay, L. B. Ware, and G. A. Zimmerman, "The acute respiratory distress syndrome," The Journal of Clinical Investigation, vol. 122, no. 8, pp. 2731-2740, 2012.

[3] M. A. Matthay and R. L. Zemans, "The acute respiratory distress syndrome: pathogenesis and treatment," Annual Review of Pathology, vol. 6, pp. 147-163, 2011.

[4] M. Chakraborty, E. P. McGreal, and S. Kotecha, "Acute lung injury in preterm newborn infants: mechanisms and 
management," Paediatric Respiratory Reviews, vol. 11, no. 3, pp. 162-170, 2010, quiz 170.

[5] J. S. Rettig, C. D. Smallwood, B. K. Walsh et al., "High-frequency oscillatory ventilation in pediatric acute lung injury: a multicenter international experience," Critical Care Medicine, vol. 43, no. 12, pp. 2660-2667, 2015.

[6] X. Pei, X. J. Zhang, and H. M. Chen, "Bardoxolone treatment alleviates lipopolysaccharide (LPS)-induced acute lung injury through suppressing inflammation and oxidative stress regulated by Nrf2 signaling," Biochemical and Biophysical Research Communications, vol. 516, no. 1, pp. 270-277, 2019.

[7] M. A. Matthay, A. Goolaerts, J. P. Howard, and J. Woo Lee, "Mesenchymal stem cells for acute lung injury: preclinical evidence," Critical Care Medicine, vol. 38, 10 Supplement, pp. S569-S573, 2010.

[8] H. Lv, Z. Yu, Y. Zheng et al., "Isovitexin exerts anti-inflammatory and anti-oxidant activities on lipopolysaccharide-induced acute lung injury by inhibiting MAPK and NF- $\kappa \mathrm{B}$ and activating HO-1/Nrf2 pathways," International Journal of Biological Sciences, vol. 12, no. 1, pp. 72-86, 2016.

[9] M. L. Franco, P. Waszak, G. Banalec et al., "LPS-induced lung injury in neonatal rats: changes in gelatinase activities and consequences on lung growth," Lung Cellular and Molecular Physiology, vol. 282, no. 3, pp. L491-L500, 2002.

[10] A. A. Kroon, J. Wang, Z. Huang, L. Cao, M. Kuliszewski, and M. Post, "Inflammatory response to oxygen and endotoxin in newborn rat lung ventilated with low tidal volume," Pediatric Research, vol. 68, no. 1, pp. 63-69, 2010.

[11] Q. Ding, G. Q. Liu, Y. Y. Zeng et al., "Role of IL-17 in LPSinduced acute lung injury: anin vivostudy," Oncotarget, vol. 8, no. 55, pp. 93704-93711, 2017.

[12] X. Wang, P. He, S. Yi, and C. Wang, "Thearubigin regulates the production of Nrf2 and alleviates LPS-induced acute lung injury in neonatal rats," Biotech, vol. 9, no. 12, p. 451, 2019.

[13] S. Becker, S. Mundandhara, R. Devlin, and M. Madden, "Regulation of cytokine production in human alveolar macrophages and airway epithelial cells in response to ambient air pollution particles: further mechanistic studies," Toxicology and Applied Pharmacology, vol. 207, no. 2, pp. 269-275, 2005.

[14] J. B. Kim, A. R. Han, E. Y. Park et al., "Inhibition of LPSinduced iNOS, COX-2 and cytokines expression by poncirin through the NF-kappaB inactivation in RAW 264.7 macrophage cells," Biological \& Pharmaceutical Bulletin, vol. 30, no. 12, pp. 2345-2351, 2007.

[15] K. Srinivasan, "Biological activities of red pepper (Capsicum annuum) and its pungent principle capsaicin: a review," Critical Reviews in Food Science and Nutrition, vol. 56, no. 9, pp. 1488-1500, 2016.

[16] M. Lu, C. Chen, Y. Lan et al., "Capsaicin-the major bioactive ingredient of chili peppers: bio-efficacy and delivery systems," Food \& Function, vol. 11, no. 4, pp. 2848-2860, 2020.

[17] P. Anandakumar, S. Kamaraj, S. Jagan et al., "Capsaicin inhibits benzo(a)pyrene-induced lung carcinogenesis in an in vivo mouse model," Inflammation Research, vol. 61, no. 11, pp. 1169-1175, 2012.

[18] Y. Xu, Q. Gu, and C. Qu, “Capsaicin pretreatment reversed pulmonary arterial hypertension by alleviating inflammation via p38MAPK pathway," Experimental Lung Research, vol. 43, no. 1, pp. 8-18, 2017.

[19] L. M. Lu, T. T. Yu, X. W. He, J. Tang, and X. W. Li, "Effect of small dose capsaicin for treatment of pulmonary fibrosis in mice and its mechanism," Zhongguo ying yong sheng li xue $z a$ zhi $=$ Zhongguo yingyong shenglixue zazhi = Chinese Journal of Applied Physiology, vol. 36, no. 3, pp. 216-222, 2020.

[20] M. Mo, S. Li, Z. Dong et al., "S-Allylmercaptocysteine ameliorates lipopolysaccharide-induced acute lung injury in mice by inhibiting inflammation and oxidative stress via nuclear factor kappa B and Keap1/Nrf2 pathways," International Immunopharmacology, vol. 81, article 106273, 2020.

[21] M. Bhatia and S. Moochhala, "Role of inflammatory mediators in the pathophysiology of acute respiratory distress syndrome," The Journal of Pathology, vol. 202, no. 2, pp. 145156, 2004.

[22] V. Fanelli and V. M. Ranieri, "Mechanisms and clinical consequences of acute lung injury," Annals of the American Thoracic Society, vol. 12, Supplement 1, pp. S3-S8, 2015.

[23] A. Sapru, H. Flori, M. W. Quasney, and M. K. Dahmer, "Pathobiology of acute respiratory distress syndrome," Pediatric Critical Care Medicine, vol. 16, 5 Supplement 1, pp. S6-22, 2015.

[24] A. Duggal, A. Ganapathy, M. Ratnapalan, and N. K. Adhikari, "Pharmacological treatments for acute respiratory distress syndrome: systematic review," Minerva Anestesiologica, vol. 81, no. 5, pp. 567-588, 2015.

[25] Y. Butt, A. Kurdowska, and T. C. Allen, "Acute lung injury: a clinical and molecular review," Archives of Pathology \& Laboratory Medicine, vol. 140, no. 4, pp. 345-350, 2016.

[26] X. Chu, K. Song, K. Xu et al., "Ceftiofur attenuates lipopolysaccharide-induced acute lung injury," International Immunopharmacology, vol. 10, no. 5, pp. 600-604, 2010.

[27] H. Seki, S. Tasaka, K. Fukunaga et al., "Effect of Toll-like receptor 4 inhibitor on LPS-induced lung injury," Inflammation Research, vol. 59, no. 10, pp. 837-845, 2010.

[28] B. V. Patel, M. R. Wilson, K. P. O’Dea, and M. Takata, “TNFinduced death signaling triggers alveolar epithelial dysfunction in acute lung injury," Journal of Immunology, vol. 190, no. 8, pp. 4274-4282, 2013.

[29] C. Pierrakos, M. Karanikolas, S. Scolletta, V. Karamouzos, and D. Velissaris, "Acute respiratory distress syndrome: pathophysiology and therapeutic options," Journal of Clinical Medicine Research, vol. 4, no. 1, pp. 7-16, 2012.

[30] P. A. Ward and J. J. Grailer, "Acute lung injury and the role of histones," Translational Respiratory Medicine, vol. 2, no. 1, 2014.

[31] V. Kumar, "Pulmonary innate immune response determines the outcome of inflammation during pneumonia and sepsisassociated acute lung injury," Frontiers in Immunology, vol. $11,2020$.

[32] J. Grommes and O. Soehnlein, "Contribution of neutrophils to acute lung Injury," Molecular Medicine, vol. 17, no. 3-4, pp. 293-307, 2011.

[33] H. Chen, C. Bai, and X. Wang, "The value of the lipopolysaccharide-induced acute lung injury model in respiratory medicine," Expert Review of Respiratory Medicine, vol. 4, no. 6, pp. 773-783, 2010.

[34] A. E. Williams and R. C. Chambers, "The mercurial nature of neutrophils: still an enigma in ARDS?," American Journal of Physiology-Lung Cellular and Molecular Physiology, vol. 306, no. 3, pp. L217-L230, 2014.

[35] R. L. Castillo, R. Carrasco Loza, and C. Romero-Dapueto, "Pathophysiological approaches of acute respiratory distress syndrome: novel bases for study of lung injury," The Open Respiratory Medicine Journal, vol. 9, pp. 83-91, 2015. 
[36] F. Wang, X. Fu, X. Wu et al., "Bone marrow derived $\mathrm{M}_{2}$ macrophages protected against lipopolysaccharide-induced acute lung injury through inhibiting oxidative stress and inflammation by modulating neutrophils and T lymphocytes responses," International Immunopharmacology, vol. 61, pp. 162-168, 2018.

[37] Y. Chen, J. Dong, J. Liu et al., "Network pharmacology-based investigation of protective mechanism of Aster tataricus on lipopolysaccharide-induced acute lung injury," International Journal of Molecular Sciences, vol. 20, no. 3, 2019.

[38] D. Togbe, S. Schnyder-Candrian, B. Schnyder et al., "Toll-like receptor and tumour necrosis factor dependent endotoxininduced acute lung injury," International Journal of Experimental Pathology, vol. 88, no. 6, pp. 387-391, 2007.

[39] S. Mukhopadhyay, J. R. Hoidal, and T. K. Mukherjee, "Role of TNF $\alpha$ in pulmonary pathophysiology," Respiratory Research, vol. 7, no. 1, 2006.

[40] A. Varelias, K. H. Gartlan, E. Kreijveld et al., "Lung parenchyma-derived IL-6 promotes IL-17A-dependent acute lung injury after allogeneic stem cell transplantation," Blood, vol. 125, no. 15, pp. 2435-2444, 2015.

[41] K. De Filippo, A. Dudeck, M. Hasenberg et al., "Mast cell and macrophage chemokines CXCL1/CXCL2 control the early stage of neutrophil recruitment during tissue inflammation," Blood, vol. 121, no. 24, pp. 4930-4937, 2013.

[42] J. Meng, Y. Zou, J. Chen et al., "sTLR4/sMD-2 complex alleviates LPS-induced acute lung injury by inhibiting proinflammatory cytokines and chemokine CXCL1 expression," Experimental and Therapeutic Medicine, vol. 16, no. 6, pp. 4632-4638, 2018.

[43] H. W. Zhang, Q. Wang, H. X. Mei et al., "RvD1 ameliorates LPS-induced acute lung injury via the suppression of neutrophil infiltration by reducing CXCL2 expression and release from resident alveolar macrophages," International Immunopharmacology, vol. 76, article 105877, 2019.

[44] Y. Xie, K. Liu, J. Luo et al., "Identification of DDX58 and CXCL10 as potential biomarkers in acute respiratory distress syndrome," DNA and Cell Biology, vol. 38, no. 12, pp. 14441451, 2019.

[45] S. Vallabhapurapu and M. Karin, "Regulation and function of NF- $\kappa \mathrm{B}$ transcription factors in the immune system," Annual Review of Immunology, vol. 27, no. 1, pp. 693-733, 2009.

[46] C. M. Gross, M. Kellner, T. Wang et al., "LPS-induced acute lung injury involves NF- $\kappa \mathrm{B}$-mediated downregulation of SOX18," American Journal of Respiratory Cell and Molecular Biology, vol. 58, no. 5, pp. 614-624, 2018.

[47] P. A. Ward, "Oxidative stress: acute and progressive lung injury," Annals of the New York Academy of Sciences, vol. 1203, pp. 53-59, 2010.

[48] H. Yang, H. Lv, H. Li, X. Ci, and L. Peng, "Oridonin protects LPS-induced acute lung injury by modulating Nrf2-mediated oxidative stress and Nrf2-independent NLRP3 and NF- $\kappa \mathrm{B}$ pathways," Cell Communication and Signaling : CCS, vol. 17, no. $1,2019$. 